\title{
Hysterosonography and Transvaginal Ultrasonography for Detection of Intrauterine Lesions in Women with Abnormal Uterine Bleeding
}

\author{
Iqbal Hussain Dogar, ${ }^{1}$ Mahjabeen Masood, ${ }^{2}$ Mahesh Gautam, ${ }^{3}$ Mahjabeen Tariq ${ }^{4}$
}

\begin{abstract}
Abnormal Uterine bleeding (AUB) is one of the frequent complaints of female patients of all ages.AUB is present in $33 \%$ of women referred to gynecologists and this increases to $69 \%$ in peri-menopausal and postmenopausal women. About $10 \%$ of postmenopausal bleeding results from endometrial cancer and imaging is the mainstay for its identification. Imaging plays a vital role in differentiating structural lesions like endometrial carcinomas, myomas and polyps which require surgical management from functional disorders requiring medical management. Transvaginal Ultrasonography (TVUS) is the first line imaging modality for AUB after selecting the patients with inconclusive pelvic ultrasonographic results. Hysterosonography (HSG) also plays a pivotal role.
\end{abstract}

\footnotetext{
${ }^{1}$ HOD/Associate Professor of Paediatric Radiology, Department of Radiodiagnosis and Medical Imaging, King Edward Medical University, Lahore.

${ }^{2}$ Assistant Professor, Department of Radiodiagnosis and Medical Imaging, King Edward Medical University, Lahore.

${ }^{3}$ Resident Department of Radiodiagnosis and Medical Imaging, King Edward Medical University, Lahore.

${ }^{4}$ Consultant Radiologist, Department of Radiodiagnosis and Medical Imaging, King Edward Medical University, Lahore.
}

\section{Date of Submission: 06-08-2016}

Date of Revision Received: 01-09-2016

Date of Acceptance for publication: 27-01-2017

Conflict of Interest: None

Funding Source: None

\section{Contribution}

All Authors have contributed in Study Design, Data Collection, Data Analysis, Data Interpretation, Manuscript Writing and Approval.
Objectives: To compares between TVUS and HSG in the detection and identification of intrauterine lesions in patients with abnormal uterine bleeding, and comparing the sensitivity and specificity of the respective methods in the detection of such lesions.

Methods: Thisstudy was conducted in Department of Radiology, Mayo Hospital Lahore. Fifty women presented with history of abnormal uterine bleeding were included in this study. Pregnancy was ruled out by transabdominal scan. Transvaginal ultrasound and hysterosonography were performed in all the patients. All the data were coded and analyzed using SPSS version 20.

Results: Out of 50 patients, 10 patients had intramyometrial fibroid, 11 had submucosal fibroid, endometrial polyp was found in 17 patients and 2 patients had thickened endometrium (thickness $>8 \mathrm{~mm}$ ). The sensitivity and specificity of TVUS was found to be $83.2 \%$ and $82.7 \%$ respectively whereas HSG showed sensitivity and specificity of $95.4 \%$ and $91.5 \%$ respectively. In the detection of the submucosal fibroid and endometrial polyp, HSG showed highest sensitivity and specificity as compared totransvaginal ultrasound.

Conclusion: Both the TVUS and HSG have comparable sensitivity and specificity in the detection of endometrial disease in patients presented with abnormal uterine bleeding however HSG is more sensitive in the detection of polyps.

Keywords: Trans vaginal ultrasound (TVUS), Hysterosonography (HSG), Endometrial polyps, Abnormal Uterine bleeding. 


\section{Introduction}

Abnormal Uterine bleeding (AUB) is one of the frequent complaints of female patients of all ages. ${ }^{1}$ AUB is present in $33 \%$ of women referred to gynecologists and this increases to $69 \%$ in peri menopausal and postmenopausal women. About $10 \%$ of postmenopausal bleeding results from endometrial cancer and imaging is the mainstay for its identification. ${ }^{2}$

In women of child bearing age, abnormal uterine bleeding includes any change in menstrual-period duration, amount or frequency and also the bleeding in between cycles. ${ }^{3}$ In postmenopausal women, abnormal uterine bleeding includes vaginal bleeding for 12 months or more after menopause. ${ }^{4}$

AUB can be due to anovulation, pregnancy related pathologies, hormonal disorders or structural lesions. The etiology may be local anatomical lesions; benign or malignant, endocrine disorders, organic disorders or iatrogenic causes. Imaging plays a vital role in differentiating structural lesions like endometrial carcinomas, myomas, polyps, atrophy etc which require surgical management from functional disorders requiring medical management. ${ }^{1}$

This study, however, will focus on the abnormal bleeding caused by intrauterine structural lesions. Transvaginal Ultrasonography (TVUS) is the first line imaging modality for AUB after selecting the patients with inconclusive pelvic ultrasonographic results. ${ }^{1}$ Hysterosonography (HSG) also plays a pivotal role and was introduced by Nannini et al. in $1981 .{ }^{5}$ With the help of a catheter, they infused sterile saline into the uterine cavity causing its expansion and thus providing an echographic contrast with the adjacent structures to allow for better visualization of the endometrium. ${ }^{5,6}$

As compared to TVUS, HSG provides better images, with a more accurate measurement of the endometrial thickness, heterogeneity and etiology of the thickness, as well as distinguishing between focal and diffuse abnormalities. ${ }^{7}$

The current study is aimed at providing a comparison between TVUS and HSG in the detection and identification of intrauterine lesions in patients with abnormal uterine bleeding, and comparing the sensitivity and specificity of the respective methods in the detection of such lesions.

\section{Methods}

This comparative study was conducted in Department of Radiology, Mayo Hospital Lahore from November
2015 to April 2016. 50 women of the age group 15 to 45 years, presented with history of abnormal uterine bleeding fulfilling the inclusion criteria were included in this study. Pregnancy was ruled out by transabdominal scan.Informed consent was taken. The age, symptoms, medical history and clinical findings of all the patients were recorded. Transvaginalscan as well as hysterosonography was performed in all patients and findings were recorded.

The patient was placed in the lithotomy position. Cervix was cleaned using piodene solution and speculum was inserted. Cervical os was localised and cleaned with piodene solution. Foleys catheter of size 5 to 7 French was inserted through the os and was inflated using $2 \mathrm{ml}$ of normal saline and speculum was removed. A standard transvaginal ultrasound was inserted alongside the catheter and 10 to $20 \mathrm{ml}$ of normal saline was instilled into the endometrial cavity.

The following variables were assessed in both TVUS and HSG. Endometrial thickness $>8 \mathrm{~mm}$, presence of submucosal fibroid, presence of intramyometrial fibroid, presence of endometrial polyp and presence of any adnexal pathology. Hypoechoic lesions altering the endometrial cavity were considered submucosal fibroid. Hypoechoic lesions in the myometrium were considered intra myometrial fibroid. Hyperechoic lesions within the endometrial cavity were considered as polyp. TVUS and HSG were performed by the single consultant radiologist. It was performed in TOSHBA Ultrasound machine using 6.5MHZ multi frequency transvaginal probe. Both the TVS and HSG were performed by consultant radiologist of Mayo Hospital. The patients were followed up after respective surgeries. The histopathological analysis of the lesions was done in the Pathology Department of Mayo Hospital, Lahore and the histopathology reports were considered as the gold standard. All the findings were collected in a predesigned proforma. The data were coded and analysed using SPSS version 20. The sensitivity, specificity, positive predictive value, negative predictive value, false positive and false negative were calculated.

\section{Results}

Out of 50 patients, 10 patienthad intra-myometrial fibroid, 11 had submucosal fibroid, endometrial polyp was found in 10 patients and 2 patients had thickened endometrium (thickness $>8 \mathrm{~mm}$ ). 
Table 1: Abnormal findings in Surgery, TVS and Hysterosonography.

\begin{tabular}{|l|c|c|}
\hline Abnormal Surgery Findings & N & \% \\
\hline Polyp & 17 & 20 \\
\hline Intramymetrial fibroid & 10 & 32 \\
\hline Submucosal fibroid & 11 & 26 \\
\hline Endometrial thickness & 2 & 22 \\
\hline Adenxal pathology & 0 & 0 \\
\hline Abnormal TVS Findings & 6 & 8 \\
\hline Polyp & 14 & 28 \\
\hline Intramyometrial fibroid & 4 & 12 \\
\hline Submucosal fibroid & 17 & 34 \\
\hline Endometrial thickness & 0 & 0 \\
\hline Adenxal pathology & \multicolumn{2}{|l|}{} \\
\hline Abnormal HSG Findings & 14 & 20 \\
\hline Polyp & 16 & 32 \\
\hline Intramymetrial fibroid & 9 & 24 \\
\hline Submucosal fibroid & 6 & 20 \\
\hline Endometrial thickness & 0 & 0 \\
\hline Adenxal pathology &
\end{tabular}

Table 2: Comparison between surgical findings and TVS and hysterosonography.

\begin{tabular}{|l|c|c|c|}
\hline \multirow{2}{*}{ TVS } & \multicolumn{3}{|c|}{ Surgical Findings } \\
\cline { 2 - 4 } & Abnormal & Normal & Total \\
\hline Abnormal & 38 & 3 & 41 \\
\hline Normal & 2 & 7 & 9 \\
\hline
\end{tabular}

\begin{tabular}{|l|r|r|r|}
\hline HSG & \multicolumn{3}{|l|}{} \\
\hline Abnormal & 38 & 2 & 43 \\
\hline Normal & 2 & 8 & 7 \\
\hline Total & 40 & 10 & 50 \\
\hline
\end{tabular}

Table 3: Comparison between Surgical Findings and TVS and Hysterosonography for Endometrial Polyps.

\begin{tabular}{|l|c|c|c|}
\hline \multirow{2}{*}{ Surgical Findings } & Present & Absent & Total \\
\cline { 2 - 4 } & $\mathbf{1 7}$ & $\mathbf{3 3}$ & $\mathbf{5 0}$ \\
\hline TVS & 5 & 1 & 6 \\
\hline Present & 12 & 32 & 44 \\
\hline Absent & \multicolumn{5}{|}{} \\
\hline HSG & 16 & 2 & 18 \\
\hline Present & 1 & 31 & 32 \\
\hline Absent & 17 & 33 & 50 \\
\hline \multicolumn{1}{|l}{ Total }
\end{tabular}

Table 4: Comparison between Surgical Findings and TVS and Hysterosonography for Submucosal Fibroids.

\begin{tabular}{|l|c|c|c|}
\hline \multirow{4}{*}{ Surgical Findings } & Present & Absent & Total \\
\cline { 2 - 4 } & $\mathbf{1 1}$ & $\mathbf{3 9}$ & $\mathbf{5 0}$ \\
\hline TVS & 3 & 1 & 4 \\
\hline Present & 8 & 38 & 46 \\
\hline Absent & \multicolumn{5}{|l}{} \\
\hline HSG & 6 & 3 & 9 \\
\hline Present & 5 & 36 & 41 \\
\hline Absent
\end{tabular}

Table 5: Sensitivity (S), specificity (E), positive predictive value (PPV), negative predictive value (NPV), false-positive (FP), false-negative (FN), value for TVUS and HSG in the diagnosis of endometrial polyps and submucosal fibroids in patients with abnormal uterine bleeding.

\begin{tabular}{|l|c|c|c|c|c|c|}
\hline \multicolumn{7}{|c|}{ Abnormal Endometrial Findings } \\
\hline & S & E & PPV & FP & NPV & FN \\
\hline TVS & $95 \%$ & $70 \%$ & $93 \%$ & $7 \%$ & $78 \%$ & $22 \%$ \\
\hline HSG & $95 \%$ & $80 \%$ & $86.6 \%$ & $13.4 \%$ & $80 \%$ & $20 \%$ \\
\hline Endometrial Polyps & \\
\hline TVS & $29.4 \%$ & $96.9 \%$ & $83.3 \%$ & $16.7 \%$ & $72.7 \%$ & $27.2 \%$ \\
\hline
\end{tabular}




\begin{tabular}{|l|l|l|l|l|l|l|}
\hline HSG & $94.1 \%$ & $93.9 \%$ & $88.8 \%$ & $11.1 \%$ & $96.8 \%$ & $3.1 \%$ \\
\hline Submucosal fibroids \\
\hline TVS & $27.2 \%$ & $97.4 \%$ & $75 \%$ & $25 \%$ & $82.6 \%$ & $17.4 \%$ \\
\hline HSG & $54.5 \%$ & $93.3 \%$ & $66.6 \%$ & $33.3 \%$ & $87.8 \%$ & $12.2 \%$ \\
\hline
\end{tabular}

\section{Discussion}

Intrauterine pathologies including fibroid and endometrial polyps are common in women of reproductive age. Imaging plays a vital role in differentiating structural lesions like endometrial carcinomas, myomas, and polyps which require surgical management from functional disorders requiring medical management. As compared to TVUS, HSG provides better images, with a more accurate measurement of the endometrial thickness, heterogeneity and etiology of the thickness, as well as distinguishing between focal and diffuse abnormalities. HSG also helps in avoiding invasive diagnostic investigations as well as provides better pre-operative evaluation of those patients who may require surgical intervention. It is relatively low in cost with better patient tolerance for the procedure as well as with no major complications. ${ }^{5,6}$

The present study is to compare the TVUS and HSG in the diagnosis of endometrial pathologies. In most of the cases, surgical findings and histopathology reports are taken as a gold standard. A single observer did all the HSG and TVUS so to eliminate the chances of inter-observer variability. ${ }^{7}$

HSG is quite effective in diagnosing endometrial abnormalities with a sensitivity of $95 \%$ and specificity of $80 \%$ similar to the results reported by other studies and also with a high NPV implying to a very less number of undiagnosed intra-cavitatory lesions.

In this study endometrial polyp was found in 17 patients, intramyometrial fibroid in 10, submucosal fibroid in 11 and thickened endometrium was found in 2 patients. Polyps were found to be the most common pathology detected in women of reproductive age period presented with the abnormal uterine bleeding. This is similar to the study conducted by Dueholm et al who reported having observed $35 \%$ of polyps or myomas in a series of 470 premenopausal patients with abnormal uterine bleeding. ${ }^{8}$ In this study, only two cases were found to be of endometrial hyperplasia which presented "normal" study in HSG and TVUS which corresponds to the study done by Ceccato Jr. et al. ${ }^{9}$

Regarding the diagnosis of polyp, HSG is found to have more sensitivity and specificity as compared to TVUS. In this study, the sensitivity and specificity of HSG in detection of endometrial polyps is found to be $94.1 \%$ and $93.9 \%$ respectively, with the respective PPV and NPV of $88.8 \%$ and $96.8 \%$. Similarly the sensitivity and specificity of TVUS is found to be $29.4 \%$ and $96.9 \%$ respectively with the respective PPV and NPV of $83.3 \%$ and $72.7 \%$. This is similar to the other studies. A study by Jacques et al found accuracy of $91.3 \%$. Similarly, Kamel et al study shows accuracy of $93.3 \%$ in the diagnosis of polyps usingHSG. ${ }^{10,11}$ Our studies also show similar results. Schwärzler et al. study in 104 patients with AUB have observed that HSG increases the diagnostic rate ofpolyps from $56 \%$ to $84 \%$, however, using HSGthe detection of neoplastic and preneoplasticconditions of the endometrium was not found to be improved. ${ }^{12}$ This is due to the reason that HSGis more accurate in diagnosis offocal than diffused lesions. Similarly the sensitivity and specificity of HSG in the detection of the submucosal fibroid is $54.5 \%$ and $93.3 \%$ respectively as compared to TVUS with the respective sensitivity and specificity of $29.4 \%$ and $96.9 \%$. Elsayes et al., observed that HSG is particularly of importance in diagnosing between anovulatory uterine bleeding and AUB due to anatomical lesions in premenopausal women and to differentiate between intracavitatory lesions and endometrial atrophy in post-menopausal women so to infer for biopsy and/or surgical management. ${ }^{6}$

Magnetic Resonance Imaging (MRI) is analternative diagnostic modalities for endometrial pathologies. It is a non-invasive procedure. It is a precise technique in diagnosis of myomas particularly when selecting the appropriate approach in removal of myomas. However, few studies shows the failure of MRI in diagnosis of endometrial polyps. ${ }^{13}$ Furthermore, it is not readily available in all the centers especially in context of developing countries.

HSG could be performed easily and it is also cost effective and could be done in an out- patient setting. Foleys catheter could be used for catheterization which is not expensive. No complications were noticed during the procedure. So, HSG has a great role in detect- 
ion of malignant and benign diseases in women of both pre and post menopausal ages who have abnormal uterine bleeding. This has also important role in asymptomatic patients with endometrial abnormalities at TVUS. HSG may be an alternative to hysteroscopy. Besides being easily available and inexpensive, it is well tolerated and could be performed easily. So it has a particular important in developing countries. However, TVUS remains the initial imaging modality for diagnosing causes of AUB in postmenopausal women due to its high sensitivity, low cost, easy availability, tolerability and effectiveness in diagnosing other intracavitatory lesions.

\section{Conclusion}

Both the TVUS and HSG has comparable sensitivity and specificity in the diagnosis of endometrial disease in patients who have abnormal uterine bleeding however in the diagnosis of polyp HSG is more sensitive. In the developing countries where hysteroscopy is not easily available, HSG may be anuseful alternative modality.

\section{References}

1. Lee SI. An imaging algorithm for evaluation of abnormal uterine bleeding: does sonohysterography play a role? Menopause. 2007; 14 (5): 823-5.

2. Mencaglia L, Perino A, Hamou J. Hysteroscopy in perimenopausal and postmenopausal women with abnormal uterine bleeding. The Journal of reproductive medicine. 1987; 32 (8): 577-82.

3. Livingstone M, Fraser IS. Mechanisms of abnormal uterine bleeding. Human reproduction update. 2002; 8 (1): 60-7.

4. Lethaby A, Farquhar C, Sarkis A, Roberts H, Jepson R, Barlow D. Hormone replacement therapy in postmenopausal women: endometrial hyperplasia and irregular bleeding. Cochrane Database Syst Rev. 2000; 2:
CD000402.

5. Nannini R, Chelo E, Branconi F, Tantini C, Scarselli G. Dynamic echohysteroscopy: a new diagnostic technique in the study of female infertility. Acta Europaea fertilitatis. 1981; 12 (2): 165-71.

6. Elsayes KM, Pandya A, Platt JF, Bude RO. Technique and diagnostic utility of saline infusion sonohysterography. International Journal of Gynecology \& Obstetrics. 2009; 105 (1): 5-9.

7. Krampl E, Bourne T, Hurlen-Solbakken H, Istre O. Transvaginal ultrasonography sonohysterography and operative hysteroscopy for the evaluation of abnormal uterine bleeding. Acta obstetricia et gynecologica Scandinavica. 2001; 80 (7): 616-22.

8. Dueholm M, Forman A, Jensen M, Laursen H, Kracht P. Transvaginal sonography combined with saline contrast sonohysterography in evaluating the uterine cavity in premenopausal patients with abnormal uterine bleeding. Ultrasound in Obstetrics and Gynecology. 2001; 18 (1): 54-61.

9. Ceccato Júnior BPV, Melo VHd, Neto L. Sonohysterography in the evaluation of the uterine cavity in postmenopausal women. Revista Brasileira de Ginecologia e Obstetrícia. 2002; 24 (8): 541-5.

10. Jacques E, Verma U, Whitted R. Accuracy of saline infusion sonohysterography in diagnosis of uterine intracavitary pathology. Obstetrics \& Gynecology. 2001; 97 (4): S18.

11. Kamel HS, Darwish AM, Abdel-Rady Mohamed S. Comparison of transvaginal ultrasonography and vaginal sonohysterography in the detection of endometrial polyps. Acta obstetricia et gynecologica Scandinavica. 2000; 79 (1): 60-4.

12. Schwärzler P, Concin H, Bösch H, Berlinger A, Wohlgenannt K, Collins W, et al. An evaluation of sonohysterography and diagnostic hysteroscopy for the assessment of intrauterine pathology. Ultrasound in Obstetrics and Gynecology. 1998; 11 (5): 337-42.

13. Brown JJ, Thurnher S, Hricak H. MR imaging of the uterus: low-signal-intensity abnormalities of the endometrium and endometrial cavity. Magnetic resonance imaging. 1990; 8 (3): 309-13. 